\title{
Validation and calibration of coupled porous-medium and free-flow problems using pore-scale resolved models
}

\author{
Iryna Rybak ${ }^{1}$ (1) . Christoph Schwarzmeier ${ }^{2}$ - Elissa Eggenweiler ${ }^{1}$. Ulrich Rüde Eu $^{2,3}$
}

Received: 14 June 2019 / Accepted: 6 August 2020 / Published online: 5 September 2020

(C) The Author(s) 2020

\begin{abstract}
The correct choice of interface conditions and effective parameters for coupled macroscale free-flow and porous-medium models is crucial for a complete mathematical description of the problem under consideration and for accurate numerical simulation of applications. We consider single-fluid-phase systems described by the Stokes-Darcy model. Different sets of coupling conditions for this model are available. However, the choice of these conditions and effective model parameters is often arbitrary. We use large-scale lattice Boltzmann simulations to validate coupling conditions by comparison of the macroscale simulations against pore-scale resolved models. We analyse three settings (lid-driven cavity over a porous bed, infiltration problem and general filtration problem) with different geometrical configurations (channelised and staggered distributions of solid grains) and different sets of interface conditions. Effective parameters for the macroscale models (permeability tensor, boundary layer constants) are computed numerically for each geometrical configuration. Numerical simulation results demonstrate the sensitivity of the coupled Stokes-Darcy problem to the location of the sharp fluid-porous interface, the effective model parameters and the interface conditions.
\end{abstract}

Keywords Stokes equations · Darcy's law · Interface conditions · Lattice Boltzmann method

Mathematics Subject Classification (2010) $68 \mathrm{~N} 99 \cdot 76 \mathrm{D} 07 \cdot 76 \mathrm{M} 50 \cdot 76 \mathrm{~S} 05$

\section{Introduction}

Coupled flow systems containing a porous-medium domain and a free-flow region appear in various environmental and technical applications, such as surface-water/groundwater flow, industrial filtration and water management in fuel cells. The interaction between the flow regions is dominated by the interface driven processes and, due to their complexity, modelling such coupled flow systems is a challenging task.

Different spatial scales can be employed to investigate coupled free-flow and porous-medium systems. At the microscale (pore scale) the pore structure is fully resolved (Fig. 1, left) and the flow in the entire fluid domain (free-flow region and pore space in the porous medium) is described by the Navier-Stokes equations with the no-

Iryna Rybak

rybak@ians.uni-stuttgart.de

Extended author information available on the last page of the article. slip condition at the boundary of the solid inclusions. However, computing the microscale flow field is infeasible for practical applications as it requires detailed information about porous-medium morphology and topography which is usually unknown. Even if information on the pore structure is available, e.g. obtained from tomographic analysis $[1,2]$ or known in advance for artificially produced composite materials (GeoDict, www.math2market.com), performing microscale simulations is computationally highly demanding.

From the macroscale perspective, the whole system is described as two different continuum flow domains (free flow, porous medium) separated by an equi-dimensional transition region or a lower-dimensional (co-dimension one) sharp interface. Different mathematical models are usually used in the two flow regions. Thus, either coupling conditions at the sharp fluid-porous interface or equations that apply at the transition zone between the two flow systems are needed [3-7]. Correct specification of these conditions is essential for a complete and accurate mathematical description of flow and transport processes in compositional systems as well as for an accurate 
Fig. 1 Schematic representation of the flow system at the microscale (left) and the macroscale (right)
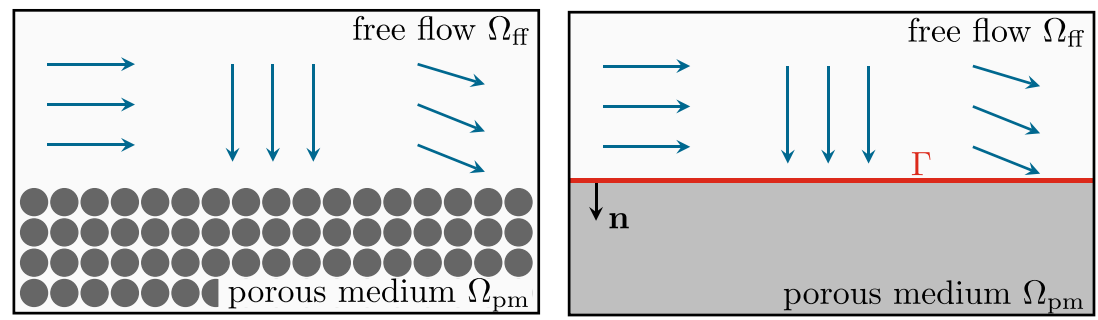

$\square$-solid phase $\square$-fluid phase $\longrightarrow$-flow direction numerical simulation of applications [8-12]. The NavierStokes equations, which in the case of low Reynolds numbers can be simplified to the Stokes equations, are typically applied to describe fluid flow in the free-flow domain, while Darcy's law or its extension is used for the flow through the porous medium. Depending on the flow regime, other models can be applied, e.g. shallow water equations or kinematic wave approximation of the Saint-Venant equation for surface flows [13-15] and the Brinkman equation, the Forchheimer equation, the Richards equation or the full two-phase porous-medium flow models for subsurface flows [16-19]. Different sets of coupling conditions are used in the literature at the fluid-porous interface [3, 5, 6, 20, 21]. Some authors distinguish between two qualitatively different flow directions: near parallel flow, where the velocity of the free fluid is much larger than the filtration velocity in the porous medium and near normal flow, where velocities in the two regions have comparable magnitudes. However, these conditions cannot be applied to a general flow situation.

The macroscopic level of flow system description is the most common one used for numerical simulations of coupled systems in practical applications [17, 18, 22-25]. Microscale (pore-scale resolved) numerical simulations are typically used to validate the macroscale models.

In this paper, we consider the Stokes-Darcy problem and analyse different coupling conditions at the fluidporous interface. Most often, the conservation of mass, the balance of normal forces and the Beavers-Joseph-Saffman condition for the tangential velocity are used both for numerical analysis and simulations (e.g. [3-5, 7, 9, 11, 12, 26]). The Beavers-Joseph-Saffman condition that describes a tangential velocity slip at the fluid-porous interface was obtained for flows parallel to the porous layer [3, 11, 27]. Nevertheless, this condition is often applied to other flow directions (e.g. [23]). Although other interface conditions are available for the Stokes-Darcy problem, they are either valid only for specific boundary value problems [20] or contain parameters which are difficult to determine [28]. There were also several attempts to validate the coupled models and the interface conditions (e.g. [29-32]). In [33] it is shown that the Beavers-Joseph condition is unsuitable for general filtration problems with arbitrary flows to the interface. However, to our knowledge no systematic study of coupling conditions has been published.

In this paper, we consider different interface conditions for the coupled Stokes-Darcy problem, different values of the Beavers-Joseph parameter, different pore geometries and flow directions, and several possible locations of the fluid-porous interface. We validate and calibrate the macroscale coupled model using pore-scale resolved numerical simulations. These fully resolved numerical simulations are performed using the lattice Boltzmann method, a numerical method for simulating fluid dynamics that is especially suited for parallel computations with high spatial and temporal resolutions in complex geometries. It thus provides first-principle insights into the physical flow processes in both domains and the flow behaviour at the interface region.

The paper is organised as follows. In Section 2 we provide the geometrical setting and the flow system description. The macroscale coupled model with different sets of interface conditions is presented in Section 3. The calculation of effective parameters for the macroscale model by means of homogenisation is discussed in Section 4 and the lattice Boltzmann method is described in Section 5 . Numerical simulation results for model validation and calibration are given in Section 6. Finally, the conclusion is presented in Section 7.

\section{Geometrical setting}

We consider a free-flow region $\Omega_{\mathrm{ff}} \subset \mathbb{R}^{2}$ and a porous medium $\Omega_{\mathrm{pm}} \subset \mathbb{R}^{2}$ with periodic arrangement of the solid obstacles (Fig. 1, left). The fluid is considered to be present in a single phase and to fully saturate the porous medium. Additionally, it is assumed to be incompressible and to have a constant viscosity. The flow is considered as being at low Reynolds numbers and supposing a single chemical 
species, no compositional effects need to be modelled. The temperature is assumed constant, such that it is not required to model any energy balance equation.

At the microscale, we solve the Stokes equations in the free-flow domain and in the pore space of the porous medium, where no-slip boundary conditions are applied on the solid-fluid boundary. At the macroscale, the system is described as two different continuum flow domains separated by the sharp interface $\Gamma$ (Fig. 1, right). We solve the Stokes and Darcy equations in the two flow domains and impose different interface conditions on the fluidporous interface (Section 3). These interface conditions are validated for different porous-medium morphologies and different flow directions.

\section{Macroscale coupled model formulation}

Fluid flow in the free-flow region is described by the Stokes equations, the flow through the porous medium is described by Darcy's law. Different sets of interface conditions are imposed on the sharp fluid-porous interface. Effective parameters such as permeability, porosity and boundary layer constants are needed to describe the porous-medium system and the interface conditions. These parameters are calculated in Section 6 for the geometrical configurations studied in the paper.

\subsection{Free-flow model}

The fluid is considered to be incompressible and therefore the mass conservation equation becomes

$\nabla \cdot \mathbf{v}=0 \quad$ in $\Omega_{\mathrm{ff}}$.

The Stokes equation describes the fluid flow

$-\nabla \cdot \mathbf{T}(\mathbf{v}, p)-\rho \mathbf{g}=0 \quad$ in $\Omega_{\mathrm{ff}}$,

where $\rho$ is the fluid density, $\mathbf{v}$ is the fluid velocity, $p$ is the pressure, $\mathbf{g}$ is the gravitational acceleration, $\mathbf{T}(\mathbf{v}, p)=$ $2 \mu \mathbf{D}(\mathbf{v})-p \mathbf{I}$ is the stress tensor, $\mu$ is the dynamic viscosity, $\mathbf{D}(\mathbf{v})=\frac{1}{2}\left(\nabla \mathbf{v}+(\nabla \mathbf{v})^{\mathrm{T}}\right)$ is the rate of strain tensor and $\mathbf{I}$ is the identity tensor.

On the external boundary of the free-flow domain $\Gamma_{\mathrm{ff}}=$ $\partial \Omega_{\mathrm{ff}} \backslash \Gamma$, the following boundary conditions are imposed

$$
\begin{aligned}
\mathbf{v} & =\overline{\mathbf{v}}_{\mathrm{ff}} & & \text { on } \Gamma_{D, \mathrm{ff}}, \\
\mathbf{T}(\mathbf{v}, p) \cdot \mathbf{n}_{\mathrm{ff}} & =\overline{\mathbf{h}}_{\mathrm{ff}} & & \text { on } \Gamma_{N, \mathrm{ff}}, \\
\partial \mathbf{v}_{\mathrm{ff}} / \partial \mathbf{n}_{\mathrm{ff}} & =0 & & \text { on } \Gamma_{\text {out }, \mathrm{ff}},
\end{aligned}
$$

where $\mathbf{n}_{\mathrm{ff}}$ is the unit outward normal vector from domain $\Omega_{\mathrm{ff}}$ on its boundary and $\Gamma_{\mathrm{ff}}=\Gamma_{D, \text { ff }} \cup \Gamma_{N \text {,ff }} \cup \Gamma_{\text {out,ff. }}$.

\subsection{Porous-medium model}

We consider Darcy's flow equations in the porous-medium domain

$\nabla \cdot \mathbf{v}=q \quad$ in $\Omega_{\mathrm{pm}}$,

where Darcy's velocity is given by

$\mathbf{v}=-\frac{\mathbf{K}}{\mu}(\nabla p-\rho \mathbf{g})$.

Here $\mathbf{v}$ is the fluid velocity through the porous medium, $p$ is the fluid pressure, $\mathbf{K}$ is the intrinsic permeability of the porous medium, $\mu$ is the dynamic viscosity of the fluid, $\rho$ is the fluid density, $q$ is the source term and $\mathbf{g}$ is the gravitational acceleration. The permeability tensor $\mathbf{K}$ is symmetric, positive definite and bounded. Its values are computed numerically for the considered settings in Section 6. In this paper, we neglect gravitational effects setting $\mathbf{g}=\mathbf{0}$.

We prescribe the following boundary conditions on the external boundary of the porous-medium domain $\Gamma_{\mathrm{pm}}=$ $\partial \Omega_{\mathrm{pm}} \backslash \Gamma:$

$$
\begin{aligned}
p=\bar{p}_{\mathrm{pm}} & \text { on } \Gamma_{D, \mathrm{pm}}, \\
\mathbf{v} \cdot \mathbf{n}_{\mathrm{pm}}=\bar{v}_{\mathrm{pm}} & \text { on } \Gamma_{N, \mathrm{pm}},
\end{aligned}
$$

where $\mathbf{n}_{\mathrm{pm}}$ is the unit outward normal vector from domain $\Omega_{\mathrm{pm}}$ on its boundary, $\Gamma_{\mathrm{pm}}=\Gamma_{D, \mathrm{pm}} \cup \Gamma_{N, \mathrm{pm}}, \Gamma_{D, \mathrm{pm}} \cap$ $\Gamma_{N, \mathrm{pm}}=\emptyset$, and $\Gamma_{D, \mathrm{pm}} \neq \emptyset$.

\subsection{Interface conditions}

In addition to the boundary conditions prescribed on the external boundary of the coupled domain, a set of interface conditions has to be defined on $\Gamma$ in order to obtain a closed problem formulation. Different sets of interface conditions have been proposed in the literature depending on the flow direction. In this paper, we validate the classical interface conditions which are typically applied to solve coupled problems and the conditions derived by homogenisation. Note that both sets of interface conditions have been developed for flows parallel to the interface, however, the classical conditions are often applied for arbitrary flows to the porous medium $[32,34]$.

\subsubsection{Classical interface conditions}

The classical set of interface conditions consists of the conservation of mass, the balance of normal forces and a variation of the Beavers-Joseph condition.

The conservation of mass across the interface $\Gamma$ is given by

$\mathbf{v}_{\mathrm{ff}} \cdot \mathbf{n}=\mathbf{v}_{\mathrm{pm}} \cdot \mathbf{n} \quad$ on $\Gamma$, 
where $\mathbf{n}$ is the unit normal vector at the fluid-porous interface $\Gamma$ pointing outward from the free-flow domain $\Omega_{\mathrm{ff}}$ (Fig. 1, right).

The balance of normal forces at the interface $\Gamma$ is

$-\mathbf{n}_{\mathrm{ff}} \cdot \mathbf{T}\left(\mathbf{v}_{\mathrm{ff}}, p_{\mathrm{ff}}\right) \cdot \mathbf{n}_{\mathrm{ff}}=p_{\mathrm{pm}} \quad$ on $\Gamma$,

where $\mathbf{n}_{\mathrm{ff}}=\mathbf{n}=-\mathbf{n}_{\mathrm{pm}}$.

There exist several possibilities for the interface condition on the tangential component of the velocity [5]. The Beavers-Joseph interface condition [3] for the difference between the tangential component of the free-flow velocity and the porous-medium velocity can be written as

$\left(\mathbf{v}_{\mathrm{ff}}-\mathbf{v}_{\mathrm{pm}}\right) \cdot \boldsymbol{\tau}_{j}+\frac{\sqrt{\mathbf{K}}}{\alpha_{\mathrm{BJ}}}\left(\nabla \mathbf{v}_{\mathrm{ff}} \cdot \mathbf{n}_{\mathrm{ff}}\right) \cdot \boldsymbol{\tau}_{j}=0 \quad$ on $\Gamma$,

where $\alpha_{\mathrm{BJ}}>0$ is the Beavers-Joseph parameter, $\boldsymbol{\tau}_{j}$ is a unit vector tangential to the interface, $j=1, \ldots, d-1$, and $d$ is the number of space dimensions.

Saffman [7] simplified the Beavers-Joseph condition (9) as follows

$\mathbf{v}_{\mathrm{ff}} \cdot \boldsymbol{\tau}_{j}+\frac{\sqrt{\mathbf{K}}}{\alpha_{\mathrm{BJ}}}\left(\nabla \mathbf{v}_{\mathrm{ff}} \cdot \mathbf{n}_{\mathrm{ff}}\right) \cdot \boldsymbol{\tau}_{j}=0 \quad$ on $\Gamma$,

arguing that the tangential velocity of a fluid through a porous medium is small and thus can be neglected.

Jones [35] considered the symmetrised rate of strain tensor $\mathbf{D}$ in the Beavers-Joseph-Saffman interface condition

$\mathbf{v}_{\mathrm{ff}} \cdot \boldsymbol{\tau}_{j}+\frac{\sqrt{\mathbf{K}}}{\alpha_{\mathrm{BJ}}} \mathbf{n}_{\mathrm{ff}} \cdot 2 \mathbf{D}\left(\mathbf{v}_{\mathrm{ff}}\right) \cdot \boldsymbol{\tau}_{j}=0 \quad$ on $\Gamma$.

\subsubsection{Interface conditions based on homogenisation}

Besides the classical interface conditions (7), (8) and (9)-(11), which have been formulated based on heuristic arguments and were justified experimentally, in [11, 27, 36] the following interface conditions were proposed

$$
\begin{aligned}
v_{\mathrm{ff}, 2}=0 & \text { on } \Gamma, \\
p_{\mathrm{ff}}+\mu C_{\omega}^{\mathrm{bl}} \frac{\partial v_{\mathrm{ff}, 1}}{\partial x_{2}}=p_{\mathrm{pm}} & \text { on } \Gamma, \\
v_{\mathrm{ff}, 1}-\varepsilon C_{1}^{\mathrm{bl}} \frac{\partial v_{\mathrm{ff}, 1}}{\partial x_{2}}=0 & \text { on } \Gamma,
\end{aligned}
$$

where $\mathbf{v}=\left(v_{1}, v_{2}\right)$ and $\varepsilon$ is the ratio of the length scales (Section 4). These coupling conditions are derived mathematically using homogenisation theory and boundary layer theory for flows parallel to the fluid-porous interface $\Gamma$. Condition (12) differs from the classical conservation of mass condition (7). However, it seems to be reasonable for flows parallel to the porous layer, because in this case the normal component of the Darcy velocity is of order $\varepsilon^{2}$ (see Eq. (20)) and thus can be neglected. The coupling condition (13) links pressure fields in the porous medium and the free-flow domain, and is a variation of the classical balance of normal forces (8). Condition (14) is a modification of the Beavers-Joseph-Saffman law [3, 7] with $\alpha_{\mathrm{BJ}}^{-1} \mathbf{K}^{1 / 2}=-\varepsilon C_{1}^{\mathrm{bl}} \mathbf{l}$. The effective coefficients $C_{1}^{\mathrm{bl}}$ and $C_{\omega}^{\mathrm{bl}}$ are determined through an auxiliary boundary layer problem. These coefficients and the parameter $\varepsilon$ are provided for different geometrical configurations in Section 6.

Remark 1 Coupling conditions for near normal flows to the porous medium are developed in [20] using the theory of homogenisation and boundary layer correctors. However, these conditions are valid only for a very specific boundary value problem and therefore cannot be applied to general flow problems.

\section{Effective properties of the coupled system}

To obtain effective properties for coupled macroscale models (effective permeability and boundary layer constants), we use the theory of homogenisation and boundary layers [11, 20, 37]. Therefore, we consider periodic porous media and assume the separation of length scales $\varepsilon=\ell / \mathcal{L} \ll 1$, where $\ell$ is the characteristic pore size and $\mathcal{L}$ is the length of the domain of interest (Fig. 2).

We consider the coupled domain $\Omega=\Omega_{\mathrm{ff}} \cup \Omega_{\mathrm{pm}}$, consisting of the free-flow region $\Omega_{\mathrm{ff}} \subset \mathbb{R}^{2}$ and the porous medium $\Omega_{\mathrm{pm}} \subset \mathbb{R}^{2}$ with periodic arrangement of the solid obstacles (Figs. 1 and 2). The flow region $\Omega^{\varepsilon}=\Omega_{\mathrm{ff}} \cup \Gamma \cup$ $\Omega_{\mathrm{pm}}^{\varepsilon}$ consists of the free-flow domain $\Omega_{\mathrm{ff}}$, the permeable interface $\Gamma$ and the pore space of the porous-medium domain $\Omega_{\mathrm{pm}}^{\varepsilon}$. The porous-medium geometry is defined by a periodic repetition of the scaled unit cell $Y=(0,1)^{2}$ in $\Omega_{\mathrm{pm}}$ (Fig. 2).

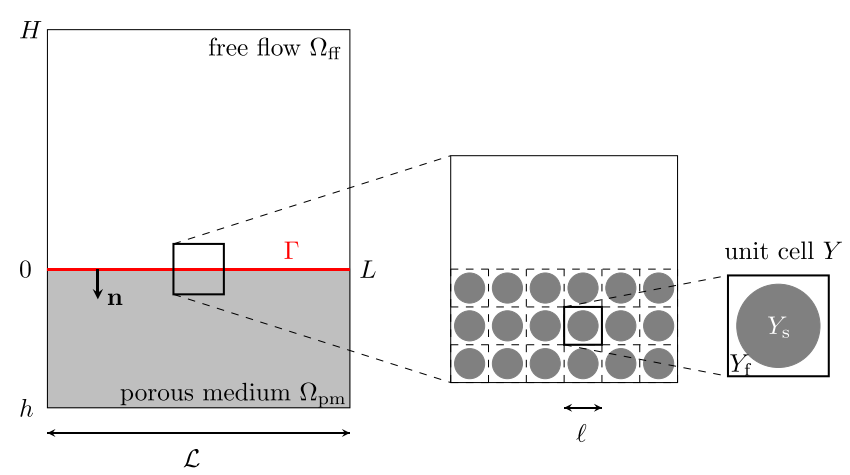

Fig. 2 Separation of scales: $\ell$-characteristic pore size, $\mathcal{L}$ macroscopic domain length 


\subsection{Microscale model}

The fluid flow in $\Omega^{\varepsilon}$ is governed by the non-dimensional steady Stokes equations

$$
\begin{aligned}
-\mu \Delta \mathbf{v}^{\varepsilon}+\nabla p^{\varepsilon} & =0 & & \text { in } \Omega^{\varepsilon}, \\
\nabla \cdot \mathbf{v}^{\varepsilon} & =0 & & \text { in } \Omega^{\varepsilon},
\end{aligned}
$$

completed with the no-slip condition on the fluid-solid interface

$\mathbf{v}^{\varepsilon}=\mathbf{0} \quad$ on $\partial \Omega^{\varepsilon} \backslash \partial \Omega$,

and the appropriate boundary conditions on the external boundary $\partial \Omega$.

For the lid-driven cavity over the porous bed described in Section 6.1, we consider

$$
\begin{array}{rlrl}
\mathbf{v}^{\varepsilon} & =(0,0) & & \text { on } \partial \Omega \backslash\left\{x_{2}=H\right\}, \\
\mathbf{v}^{\varepsilon}=(1,0) & & \text { on }\left\{x_{2}=H\right\} .
\end{array}
$$

For the infiltration problem validated in Section 6.2, we have

$$
\begin{array}{ll}
\mathbf{v}^{\varepsilon}=(0,0) & \text { on }\left\{x_{1}=0\right\} \cup\left\{x_{1}=L\right\}, \\
\mathbf{v}^{\varepsilon}=\left(0,-0.1 \sin \left(\pi x_{1}\right)\right) & \text { on }\left\{x_{2}=H\right\}, \\
\left(\nabla \mathbf{v}^{\varepsilon}-p^{\varepsilon} \mathbf{I}\right) \mathbf{n}=-p_{b} \mathbf{n} & \text { on }\left\{x_{2}=h\right\},
\end{array}
$$

where $p_{b}=100$.

For the general filtration problem with arbitrary flow direction presented in Section 6.3, we apply the following boundary conditions

$$
\begin{array}{ll}
\mathbf{v}^{\varepsilon}=\left(0,-0.2 \sin \left(\pi x_{1}\right)\right) & \text { on }\left\{x_{2}=H\right\}, \\
\left(\nabla \mathbf{v}^{\varepsilon}-p^{\varepsilon} \mathbf{I}\right) \mathbf{n}=\mathbf{0} & \text { on } \Gamma_{\text {r,out }} \cup \Gamma_{\mathrm{l}, \text { out }}, \\
\mathbf{v}^{\varepsilon}=(0,0) & \text { on } \Gamma_{\text {wall }}, \\
\left(\nabla \mathbf{v}^{\varepsilon}-p^{\varepsilon} \mathbf{I}\right) \mathbf{n}=-p_{b} \mathbf{n} & \text { on }\left\{x_{2}=h\right\},
\end{array}
$$

where $\Gamma_{1, \text { out }}=\{0\} \times(0,0.2 H), \Gamma_{\mathrm{r}, \text { out }}=\{L\} \times$ $(0, H), \Gamma_{\text {wall }}=\left(\left\{x_{1}=0\right\} \cup\left\{x_{1}=L\right\}\right) \backslash\left(\Gamma_{\mathrm{l}, \text { out }} \cup \Gamma_{\text {r,out }}\right)$ and $p_{b}=\left(10^{-6}-x_{1}\right)$.

\subsection{Homogenisation}

We obtain the corresponding macroscale formulation of the coupled system by studying the behaviour of the solutions to the microscopic problem (15), (16) with the corresponding set of boundary conditions when $\varepsilon \rightarrow 0$. In the limit, the equations in the free-flow region $\Omega_{\mathrm{ff}}$ remain unchanged and we need to homogenise the porous region. To derive the limit problem in $\Omega_{\mathrm{pm}}^{\varepsilon}$, we use the idea of homogenisation with two-scale asymptotic expansions [37, chap. 1 and 3]:

$$
\mathbf{v}^{\varepsilon}(\mathbf{x})=\varepsilon^{2} \mathbf{v}_{0}(\mathbf{x}, \mathbf{y})+\varepsilon^{3} \mathbf{v}_{1}(\mathbf{x}, \mathbf{y})+\ldots
$$$$
p^{\varepsilon}(\mathbf{x})=p_{0}(\mathbf{x}, \mathbf{y})+\varepsilon p_{1}(\mathbf{x}, \mathbf{y})+\ldots
$$

where $\mathbf{y}=\mathbf{x} / \varepsilon$. We follow the classical procedure of homogenisation to derive Darcy's law (5) as the upscaled problem in $\Omega_{\mathrm{pm}}$ completed by the appropriate boundary conditions.
The permeability tensor $\tilde{\mathbf{K}}$ is given by

$\tilde{\mathbf{K}}=\left(\widetilde{k}_{i j}\right)_{i, j=1,2}=\int_{Y_{\mathrm{f}}} w_{i}^{j} \mathrm{~d} \mathbf{y}$,

where $\mathbf{w}^{j}=\left(w_{1}^{j}, w_{2}^{j}\right)$ and $\pi^{j}$ are the solutions to the following cell problems for $j=1,2$ :

$$
\begin{array}{rlrl}
-\Delta_{\mathbf{y}} \mathbf{w}^{j}+\nabla_{\mathbf{y}} \pi^{j} & =\mathbf{e}_{j} & & \text { in } Y_{\mathrm{f}}, \quad \int_{Y_{\mathrm{f}}} \pi^{j} \mathrm{~d} \mathbf{y}=0, \\
\nabla_{\mathbf{y}} \cdot \mathbf{w}^{j} & =0 & & \text { in } Y_{\mathrm{f}}, \\
\mathbf{w}^{j} & =0 & & \text { on } \partial Y_{\mathrm{s}}, \\
\left\{\mathbf{w}^{j}, \pi^{j}\right\} \text { is 1-periodic in } \mathbf{y} . & &
\end{array}
$$

The unit cell $Y$ is presented in Fig. 2. To obtain the effective permeability $\mathbf{K}$, the tensor $\tilde{\mathbf{K}}$ has to be scaled with $\varepsilon^{2}$.

\section{Lattice Boltzmann method}

The lattice Boltzmann method (LBM) is a modern approach for simulating fluid dynamics. While the LBM historically evolved from lattice gas automata [38], it can be regarded as a special discretisation of the continuous Boltzmann equation [39] that describes the dynamics of a gas on a mesoscopic scale. On a macroscopic scale, however, the Boltzmann equation leads to the equations of fluid dynamics, i.e. mass, momentum and energy conservation. Therefore, by solving the Boltzmann equation for certain scenarios, one can also obtain the corresponding solutions to the Navier-Stokes equations [40].

\subsection{Advantages}

In comparison with conventional methods for simulating fluid dynamics, such as the finite difference, finite volume or finite element method, the LBM's main advantages lay in its relative ease of parallelisation and its suitability for complex flow scenarios. As the most compute-intensive operations are local, i.e. restricted to neighbouring lattice cells, the LBM is inherently applicable for massively parallel computing. Furthermore, the LBM is well-suited for simulating particulate flows [41, 42], multi-phase flows [43] and flows through complicated geometries such as porous media $[44,45]$.

\subsection{Description}

Our introduction to the LBM follows [40]; the interested reader is referred to this reference and the references therein. The LBM's most fundamental quantities are the so-called particle distribution functions $f_{i}(\mathbf{x}, t)$ that represent the density of virtual particles with velocity $\mathbf{c}_{i}=\left(c_{i x}, c_{i y}\right)$ at position $\mathbf{x}$ and time $t$. The index $i$ in $f_{i}$ refers to the corresponding index of velocity $\mathbf{c}_{i}$ in a discrete set of 
velocities $\left\{\mathbf{c}_{i}, w_{i}\right\}$ with weighting coefficients $w_{i}$. Velocity sets are denoted as $\mathrm{D} d \mathrm{Q} q$ with $d$ being the number of spatial dimensions and $q$ referring to the velocity set's number of velocities such that $i \in\{0,1, \ldots, q-1\}$. Velocity sets are a trade-off between accuracy and memory consumption. Different velocity sets and their corresponding coefficients can be found in the LBM literature such as [40]. Here we limit ourselves to two spatial dimensions such that we use the well-established D2Q9 velocity set. In each velocity set, the constant $c_{s}$ determines the relation between the fluid pressure $p$ and the fluid density $\rho$ as $p=c_{s}^{2} \rho$. Due to this relation, $c_{s}$ is called the lattice speed of sound. In most velocity sets used to solve the Navier-Stokes equations, it can be expressed as $c_{s}=\sqrt{1 / 3} \Delta x / \Delta t$.

The distribution function $f_{i}$ is only defined at discrete points $\mathbf{x}$ in a square lattice with lattice spacing $\Delta x$. Since the LBM's origins lay in lattice gas automata, these discrete points are also often referred to as lattice cells. Similarly, $f_{i}$ is only defined at discrete times $t$ with time step $\Delta t$. As it is common in the LBM literature, we use lattice units which is an artificial set of units that is scaled such that the relations $\Delta x=1$ and $\Delta t=1$ hold.

In kinetic theory, one obtains the macroscopic mass and momentum density by taking the moments of the Boltzmann equation's distribution functions. Similarly, one can find the mass density $\rho$ and the momentum density $\rho \mathbf{v}$ with fluid velocity $\mathbf{v}$ by

$\rho(\mathbf{x}, t)=\sum_{i} f_{i}(\mathbf{x}, t), \quad \rho \mathbf{v}(\mathbf{x}, t)=\sum_{i} \mathbf{c}_{i} f_{i}(\mathbf{x}, t)$.

The lattice Boltzmann equation

$f_{i}\left(\mathbf{x}+\mathbf{c}_{i} \Delta t, t+\Delta t\right)=f_{i}(\mathbf{x}, t)+\Theta_{i}(\mathbf{x}, t)$

describes the advection of particles $f_{i}(\mathbf{x}, t)$ that travel with velocity $\mathbf{c}_{i}$ to a neighbouring lattice cell $\mathbf{x}+\mathbf{c}_{i} \Delta t$ in the next time step $t+\Delta t$ (Fig. 3, left). After having moved from one lattice cell to another, the particles are affected by a collision operator $\Theta_{i}(\mathbf{x}, t)$ that models particle collisions by redistributing particles among $f_{i}$ (Fig. 3, right).

The simplest collision operator that can be used for solving the Navier-Stokes equations is the BhatnagarGross-Krook (BGK) operator. It linearly relaxes particle distributions towards an equilibrium $f_{i}^{\text {eq }}$ by

$\Theta_{i}(\mathbf{x}, t)=-\frac{f_{i}(\mathbf{x}, t)-f_{i}(\mathbf{x}, t)^{\mathrm{eq}}}{\tau} \Delta t$,

with a relaxation rate determined by the relaxation time $\tau$. In contrast to other collision operators, the BGK operator is based on only one relaxation time and it is therefore also called single-relaxation-time (SRT) collision operator. Due to its simplicity, the BGK collision operator is often the standard choice for LBM simulations. However, in [46], it was found to be subject to a non-physical dependence between the fluid viscosity and the boundary
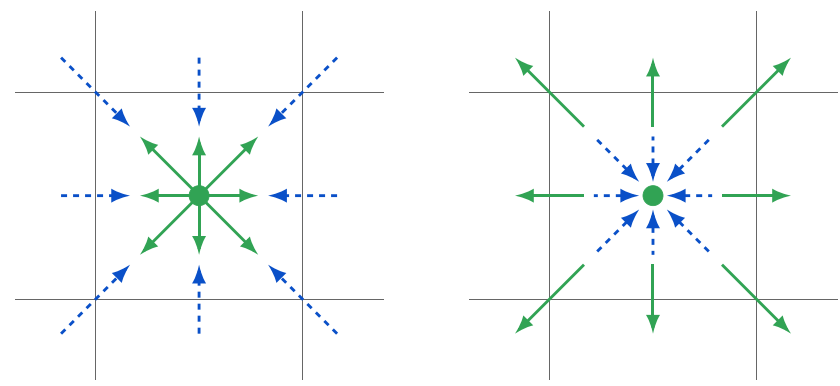

Fig. 3 Schematic representation of the lattice Boltzmann streaming and collision step for the D2Q9 velocity set. After particles have collided, they are streamed to neighbouring lattice cells (left). After streaming to neighbouring lattice cells, another particle collision occurs (right)

locations, which is especially problematic in scenarios with large boundary areas such as flow through porous media. Therefore, for the LBM simulations in this paper, we use the more accurate two-relaxation-time (TRT) collision operator [47, 48].

The particle distribution functions' equilibria

$f_{i}^{\mathrm{eq}}(\mathbf{x}, t)=w_{i} \rho\left(1+\frac{\mathbf{v} \cdot \mathbf{c}_{i}}{c_{\mathrm{s}}^{2}}+\frac{\left(\mathbf{v} \cdot \mathbf{c}_{i}\right)^{2}}{2 c_{\mathrm{s}}^{4}}-\frac{\mathbf{v} \cdot \mathbf{v}}{2 c_{\mathrm{s}}^{2}}\right)$

are derived by a Hermite polynomial expansion of the continuous Maxwell-Boltzmann distribution [40]. The moments of $f_{i}^{\mathrm{eq}}$ are identical to those of the corresponding $f_{i}$, i.e. $\Sigma_{i} f_{i}^{\mathrm{eq}}=\Sigma_{i} f_{i}=\rho$ and $\Sigma_{i} \mathbf{c}_{i} f_{i}^{\mathrm{eq}}=\Sigma_{i} \mathbf{c}_{i} f_{i}=\rho \mathbf{v}$. Since $f_{i}^{\text {eq }}$ only depends on the local quantities density $\rho$ and fluid velocity $\mathbf{v}$ (see Eq. (23)), global information exchange is not needed and the LBM is therefore well-suited for parallel computing.

Using the Chapman-Enskog analysis [40], one can show that the lattice Boltzmann equation (24) has a macroscopic behaviour that converges asymptotically to the solutions of the Navier-Stokes equations. The fluid's kinematic shear viscosity $v$ is then a function of the relaxation time $\tau$ and obtained by

$v=c_{\mathrm{s}}^{2}\left(\tau-\frac{\Delta t}{2}\right)$.

\section{Model validation and calibration}

In this section, we validate and calibrate coupled StokesDarcy models using pore-scale resolved simulations for different geometrical configurations and different flow regimes.

We study three test cases: one with flow nearly parallel to the interface (Section 6.1), one with flow perpendicular to the porous medium (Section 6.2) and one with arbitrary flow to the fluid-porous interface (Section 6.3). For the first two test cases (lid-driven cavity over a porous bed and 
infiltration), we consider the free-flow region $\Omega_{\mathrm{ff}}=[0,1] \times$ $[0,1]$ and the porous medium $\Omega_{\mathrm{pm}}=[0,1] \times[-0.5,0]$. For the general filtration problem with arbitrary flow direction we take $\Omega_{\mathrm{ff}}=[0,1] \times[0,0.5]$ and $\Omega_{\mathrm{pm}}=[0,1] \times[-0.5,0]$. The porous medium is set to be periodic for all test cases such that we can compute the effective parameters easily using homogenisation theory.

We solve the unit cell problems (22) and compute the effective permeability tensor for different geometrical settings according to Eq. (21). To compute the boundary layer constants, we solve the Stokes problem in a perforated vertical cutoff stripe containing unit cells (see, e.g., [20]). To compute the effective parameters numerically, we use the software package FrEEFEM++ with P2/P1 finite elements [49]. The dynamic viscosity of the fluid is scaled for all test cases to $\mu=1$.

The macroscale problem is discretised using the finite volume method on staggered grids. The computational domains $\Omega_{\mathrm{ff}}$ and $\Omega_{\mathrm{pm}}$ are partitioned into equal blocks with grid size $h=1 / 800$, and the grids are conforming at the interface.

We apply large-scale simulations using the LBM described in Section 5 to compute the microscale (porescale) velocity field and compare the macroscale simulations with different sets of interface conditions against these pore-scale resolved models.

The LBM simulations are implemented and performed with the open-source software-framework WALBERLA [5052] (www.walberla.net). We use the D2Q9 velocity set and the TRT collision operator. The stability and truncation errors of the simulations can be controlled with the TRT collision operator's two relaxation times. While we have tested different parameters, we found the accuracy of our simulations to be insensitive to the specific choice of these parameters. For the results that are shown in this paper, we set the even order relaxation time $\tau=$ 1 and choose the odd order relaxation time using the so-called magic parameter $\Lambda$. It was found that $\Lambda=$ $3 / 16$ exactly reproduces the parabolic flow profile in a Poiseuille channel, the walls of which are modelled by LBM bounce back boundary conditions [47, 48]. Due to the solid inclusions, the computational domain contains a significant amount of walls that are modelled with bounce back boundary conditions. We therefore regard $\Lambda=3 / 16$ a reasonable choice. With Eq. (27), the fluid's kinematic viscosity in lattice units becomes $v=1 / 6$. We consider the simulation to have reached steady state when the relative temporal change in the absolute value of the fluid's maximum velocity in $x_{2}$-direction is below $10^{-4}$.

In the following sections, we analyse the sensitivity of the coupled Stokes-Darcy models to the location of the fluidporous interface $\Gamma$, the choice of the effective parameters and the interface conditions.

\subsection{Lid-driven cavity over the porous bed}

For the lid-driven cavity problem, we consider two geometrical configurations, a channelised one schematically presented in Fig. 4 and a staggered arrangement of the solid inclusions presented in Fig. 13. In both cases there are 40 solid inclusions in $x_{1}$-direction and 20 inclusions in $x_{2}$ direction in the porous domain $\Omega_{\mathrm{pm}}$ and the porosity is $\phi=$ 0.4 . The radius of solid inclusions is $r=\frac{1}{40} \sqrt{(1-\phi) / \pi}$. We define the Reynolds number

$\operatorname{Re}=\frac{v_{\text {lid }} \mathcal{L}}{v}$

using the $x_{1}$-component of the velocity at the lid $v_{\text {lid }}$, the length of the domain $\mathcal{L}$ in $x_{1}$-direction (Fig. 2) and the kinematic fluid viscosity $v$.

\subsubsection{Channelised geometrical configuration}

In this section, we consider the channelised geometrical configuration (Fig. 4). For this geometry, we solve the cell problems (22), compute the permeability tensor according to (21) and obtain

$\tilde{\mathbf{K}}=\left(\begin{array}{cc}5.671 \cdot 10^{-4} & 0 \\ 0 & 5.671 \cdot 10^{-4}\end{array}\right)$.

The effective permeability tensor of the porous medium is given by $\mathbf{K}=\varepsilon^{2} \widetilde{\mathbf{K}}$, where $\varepsilon=1 / 40$.

The corresponding macroscale model (1), (2), (4), (5) is closed by the following boundary conditions on the external boundary

$$
\begin{array}{ll}
\mathbf{v}_{\mathrm{ff}}=(1,0) & \text { on }\left\{x_{2}=1\right\}, \\
\mathbf{v}_{\mathrm{ff}}=(0,0) & \text { on }(\{0\} \cup\{1\}) \times(0,1), \\
\mathbf{v}_{\mathrm{pm}} \cdot \mathbf{n}_{\mathrm{pm}}=0 & \text { on }\left\{x_{2}=-0.5\right\}, \\
\mathbf{v}_{\mathrm{pm}} \cdot \mathbf{n}_{\mathrm{pm}}=0 & \text { on }(\{0\} \cup\{1\}) \times(-0.5,
\end{array}
$$

In the LBM simulation, the whole domain is resolved by $2400 \times 3600$ lattice cells such that a solid inclusion has a

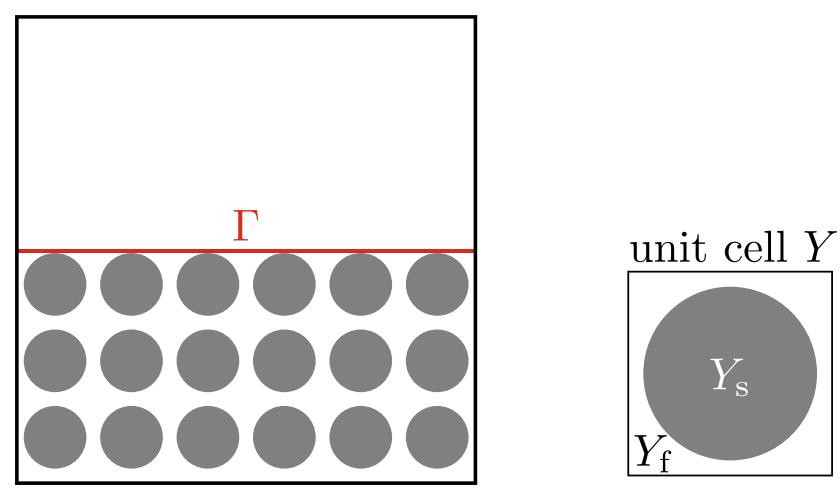

Fig. 4 Schematic illustration of the microscale setting and the location of the sharp fluid-porous interface $\Gamma$ (left) and the unit cell (right) for the channelised lid-driven cavity problem and the general filtration problem 
diameter of about 52 lattice cells. We apply simple bounce back boundary conditions [40] at the solid inclusions and at the external boundaries of the domain with respect to Eq. (30). In order to approximate Stokes flow, we set $R e=0.01$.

Two sets of interface conditions are analysed for the macroscale problem: (i) the classical conditions (7), (8), (11) and (ii) the interface conditions derived using homogenisation (12)-(14).

\section{(i) Classical interface conditions}

In this section, we study the sensitivity of the coupled macroscale Stokes-Darcy (SD) model (1), (2), (4), (5) with the classical interface conditions (7), (8), (11) to the location of the sharp fluid-porous interface and to the BeaversJoseph parameter $\alpha_{\mathrm{BJ}}$. In condition (11) we use $\sqrt{\mathbf{K}}=$ $\sqrt{\left(\mathbf{K} \boldsymbol{\tau}_{j}\right) \cdot \boldsymbol{\tau}_{j}}$.

We evaluate the cross-sections at $x_{1}=0.4875$ (intersecting solid inclusions) and $x_{1}=0.5$ (between solid inclusions), where the flow is almost parallel to the interface and near the boundary at $x_{1}=0.025$, where the flow is almost perpendicular. The interface locations for the macroscale model are $x_{2}=-0.01(s=b), x_{2}=0(s=0)$ and $x_{2}=0.01(s=t)$.

Velocity profiles ( $x_{1}$ - and $x_{2}$-components) in and close to the horizontal centre of the domain are presented in Figs. 5 and 6. The Beavers-Joseph parameter is taken $\alpha_{\mathrm{BJ}}=0.5$ and different interface locations are considered. The profiles differ in the near-interface region and the best fit of the macroscale model to the pore-scale resolved simulations is obtained for $s=0$. This means that the sharp fluid-porous

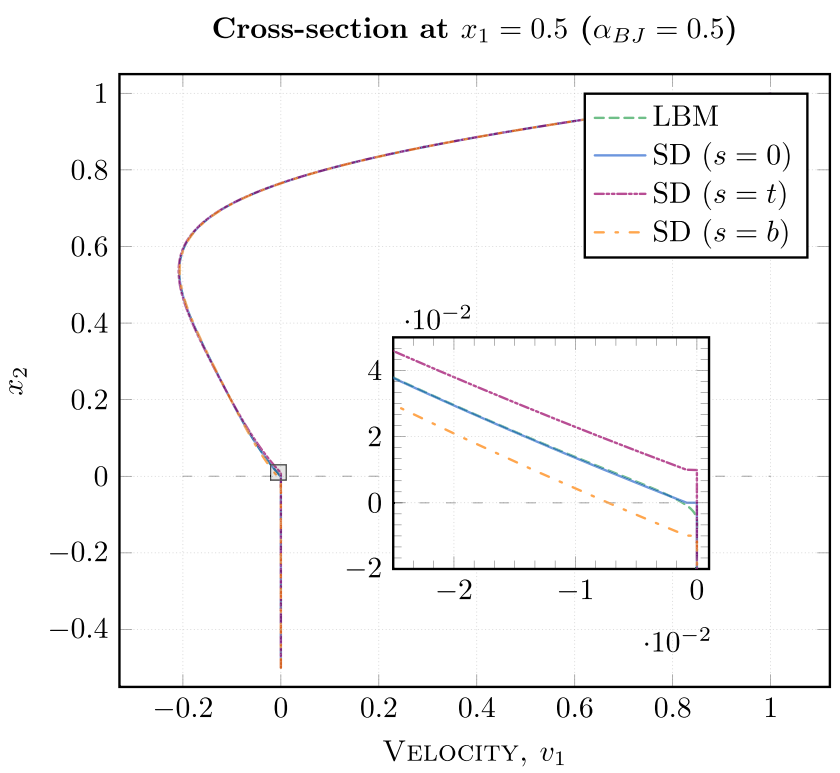

Fig. 5 Velocity profiles ( $x_{1}$-component) for the lid-driven case at $x_{1}=$ 0.5 for different interface locations zoomed near the interface

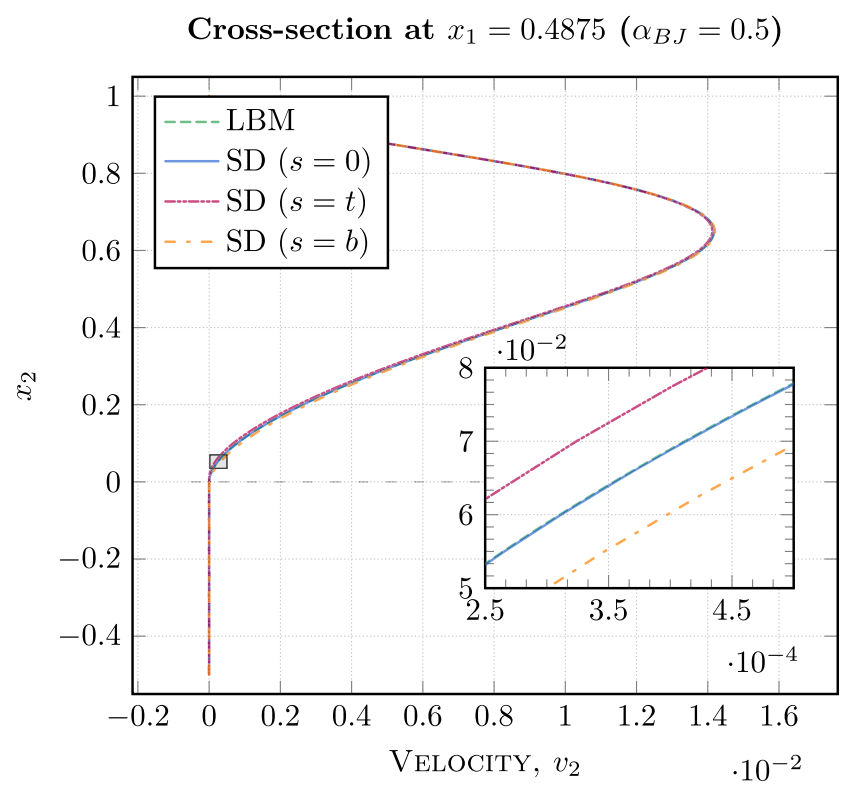

Fig. 6 Velocity profiles ( $x_{2}$-component) for the lid-driven case at $x_{1}=$ 0.4875 for different interface locations zoomed near the interface

interface $\Gamma$ should be placed directly on top of the first row of solid inclusions (Fig. 4).

Figure 7 provides velocity profiles for different values of the Beavers-Joseph parameter $\alpha_{\mathrm{BJ}}$. The best fit is obtained for $\alpha_{\mathrm{BJ}}=0.5$. Note that in the literature, $\alpha_{\mathrm{BJ}}=1$ is usually used, which is not always the optimal choice.

Velocity profiles near the left boundary are provided in Figs. 8 and 9. Here, the location of the sharp interface and also the value of the Beavers-Joseph parameter $\alpha_{\mathrm{BJ}}$ has almost no effect on the $x_{1}$-component of the velocity

$$
\text { Cross-section at } x_{1}=0.5(s=0)
$$

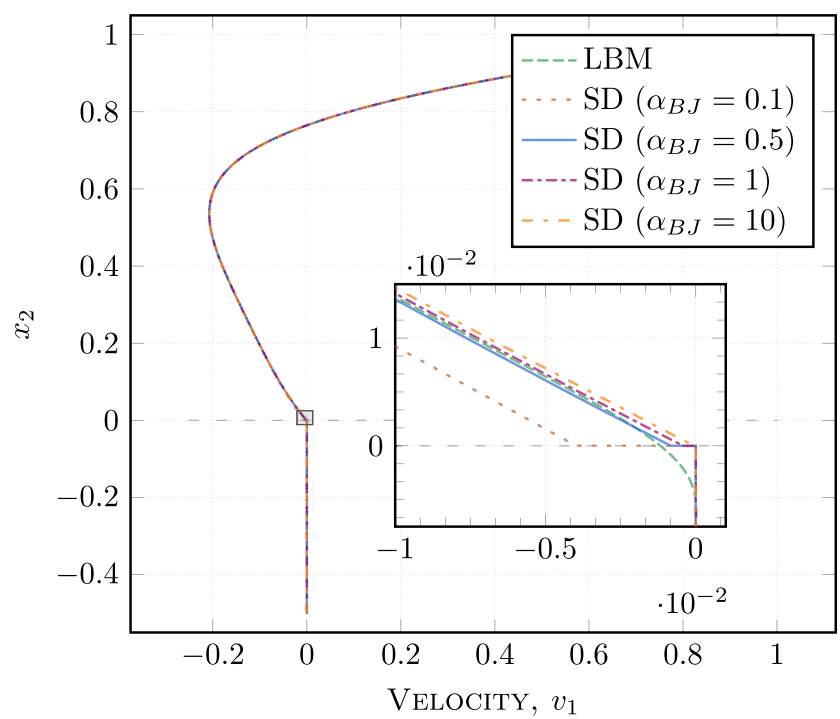

Fig. 7 Velocity profiles ( $x_{1}$-component) for the lid-driven case at $x_{1}=$ 0.5 for different values of the Beavers-Joseph coefficient zoomed near the interface 
Cross-section at $x_{1}=0.025$

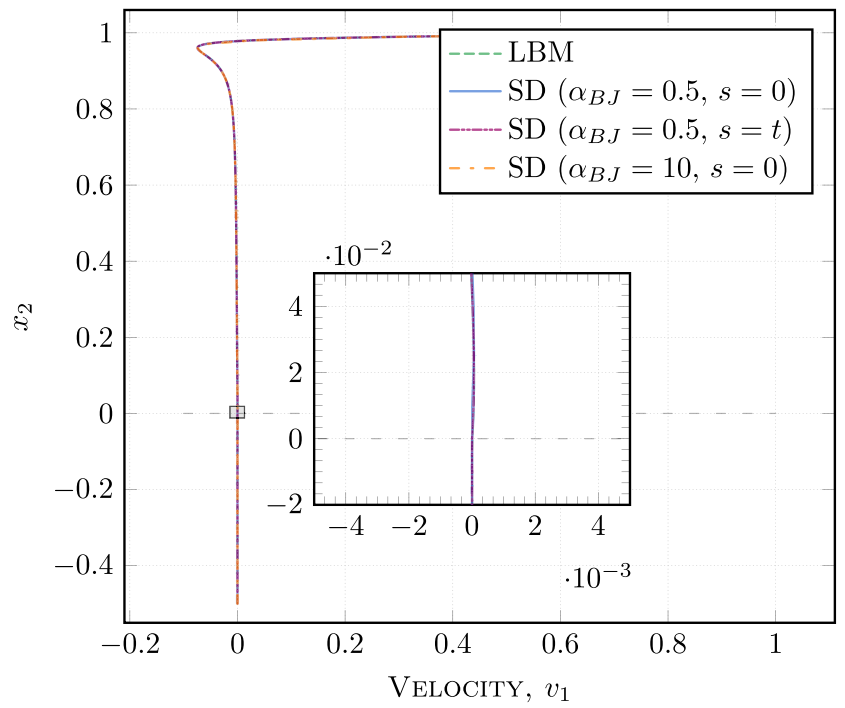

Fig. 8 Velocity profiles ( $x_{1}$-component) for the lid-driven case at $x_{1}=$ 0.025 zoomed near the interface

(Fig. 8). This is due to the fact that the flow is almost perpendicular to the porous layer and the magnitude of the horizontal velocity is very small. However, for the vertical component of the velocity, the sharp interface location is important (Fig. 9).

To demonstrate the sensitivity of the coupled StokesDarcy model to the choice of the Beavers-Joseph parameter $\alpha_{\mathrm{BJ}}$ in Eq. (11), we make cross-sections of the horizontal component of the velocity at the fluid-porous interface $\left(x_{2}=\right.$ 0 ). The numerically simulated free-flow velocity at the interface for different values of $\alpha_{\mathrm{BJ}}$ is presented in Fig. 10.

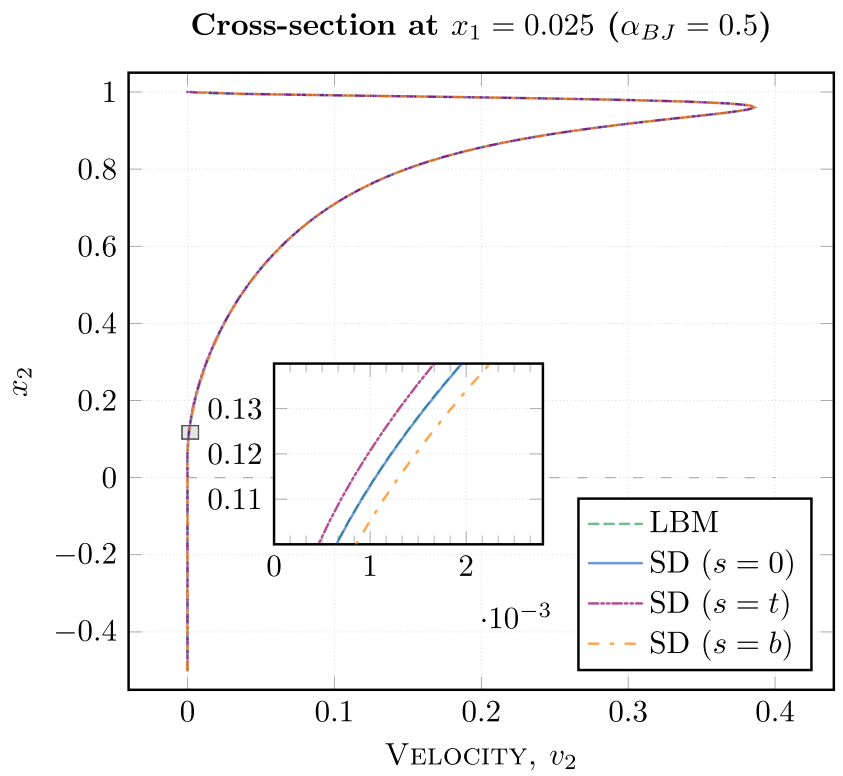

Fig. 9 Velocity profiles ( $x_{2}$-component) for the lid-driven case at $x_{1}=$ 0.025 for different interface locations zoomed near the interface

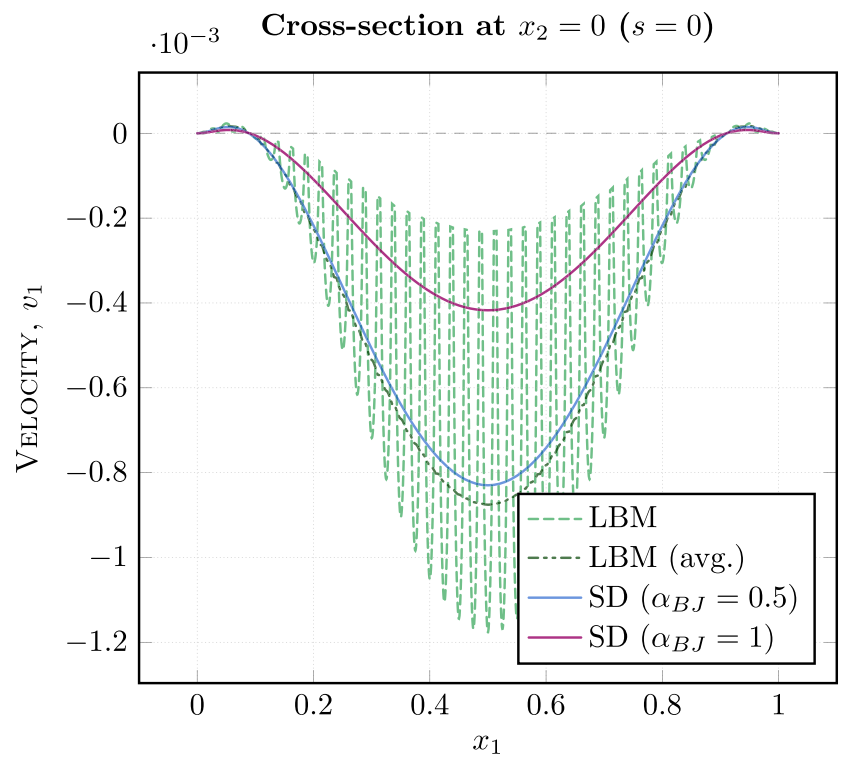

Fig. 10 Velocity profiles ( $x_{1}$-component) for the lid-driven case at the sharp fluid-porous interface $\left(x_{2}=0\right)$ for different values of the Beavers-Joseph coefficient $\alpha_{\mathrm{BJ}}$

The velocity profile of the LBM simulations fluctuates since the flow field is influenced by individual solid inclusions at the interface. In order to obtain a meaningful basis for comparison, the velocity at the interface is averaged for the pore-scale simulations. We have found that a simple unweighted moving average of 50 values, i.e. lattice cells, filters the low- and high-frequency components of the velocity very well. The width of the moving average also corresponds roughly to the solid inclusions' diameter of 52 lattice cells. For averaging the 25 values closest to the boundaries at $x_{1}=0$ and $x_{1}=1$, we assume the missing values outside the boundary to be zero. The averaged results of the pore-scale simulations suggest that the choice $\alpha_{\mathrm{BJ}}=$ 1 which is commonly used in the literature is not optimal. Again, we observed that the simulation result with $\alpha_{\mathrm{BJ}}=$ 0.5 provides a much better fit (Fig. 10).

Note, that since the Beavers-Joseph-Saffman condition is the interface condition for the horizontal component of the velocity, the vertical component is almost insensitive to a change in the value of the Beavers-Joseph parameter $\alpha_{\mathrm{BJ}}$.

\section{(ii) Interface conditions based on homogenisation}

In this section, we study the Stokes-Darcy model (1), (2), (4), (5) with the interface conditions (12)(14) proposed in $[11,36]$. To apply these interface conditions we need the boundary layer constants for the considered geometry (Fig. 4). To compute these constants, we consider a cutoff stripe in which the interface can be positioned at any distance of order $\mathcal{O}(\varepsilon)$ from the first row of solid inclusions [36]. A change of the 
Cross-section at $x_{1}=0.5$

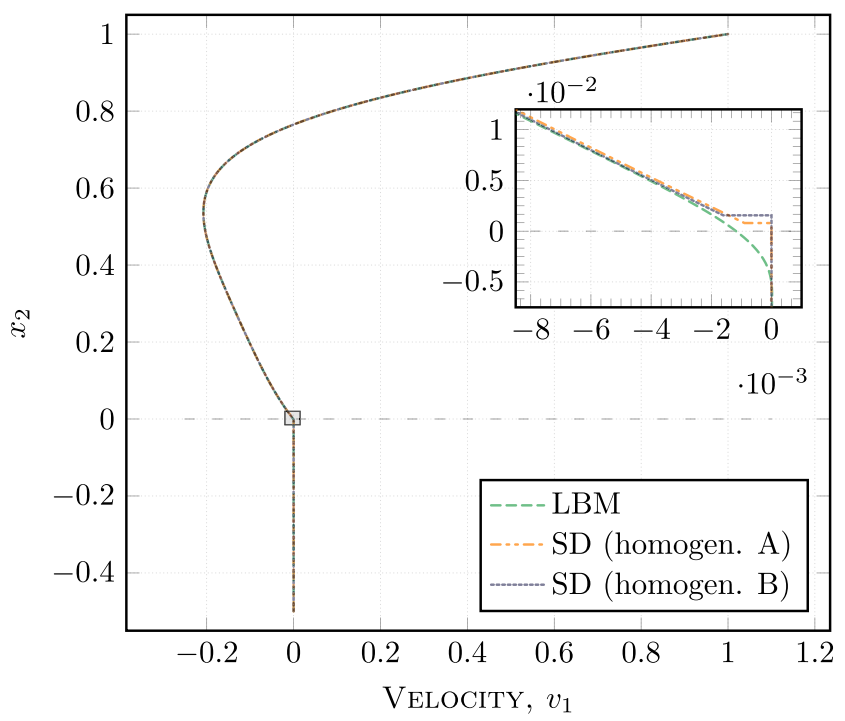

Fig. 11 Velocity profiles ( $x_{1}$-component) for the lid-driven case at $x_{1}=0.5$ for the interface conditions derived via homogenisation

interface position leads to a change of the boundary layer constants $C_{1}^{\mathrm{bl}}$ and $C_{\omega}^{\mathrm{bl}}$. Therefore, instead of finding an optimal interface location one has to compute the boundary layer constants corresponding to the chosen interface position. To demonstrate this, we consider two different interface locations, compute the corresponding boundary layer constants and validate the Stokes-Darcy model.

(A) For the interface $\Gamma$ located at $x_{2}=8.0 \cdot 10^{-4}$ we obtain the following boundary layer constants

$C_{1}^{\mathrm{bl}}=-5.13 \cdot 10^{-2}, \quad C_{\omega}^{\mathrm{bl}}=0$.

\section{Cross-section at $x_{1}=0.4875$}

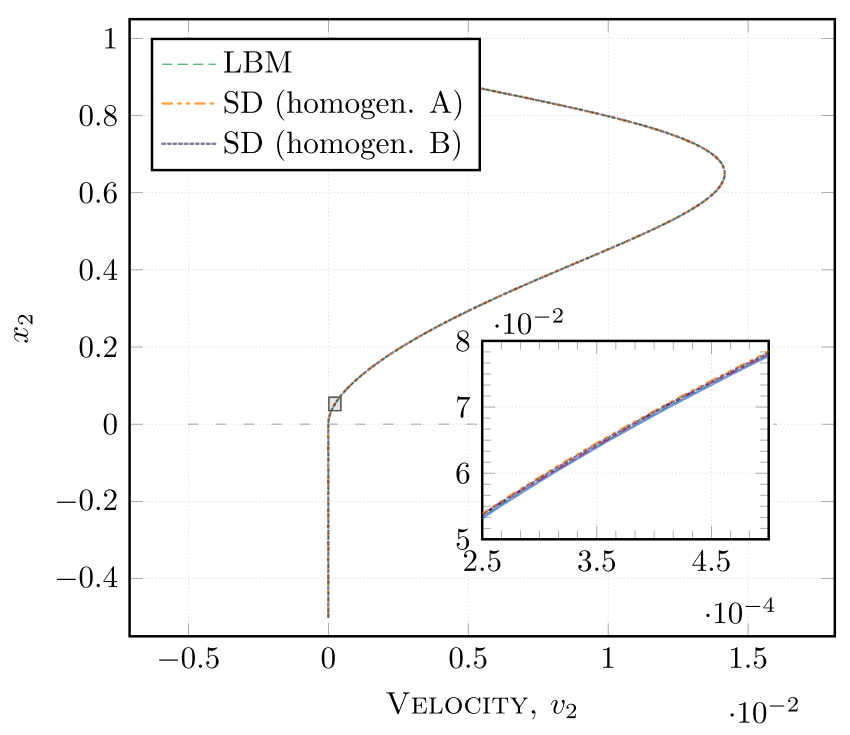

Fig. 12 Velocity profiles ( $x_{2}$-component) for the lid-driven case at $x_{1}=0.4875$ for the interface conditions derived via homogenisation
(B) For the interface $\Gamma$ located at $x_{2}=1.5625 \cdot 10^{-3}$ we obtain

$C_{1}^{\mathrm{bl}}=-9.23 \cdot 10^{-2}, \quad C_{\omega}^{\mathrm{bl}}=0$.

In Figs. 11 and 12 we provide velocity profiles for the liddriven cavity problem in and close to the horizontal centre of the domain. For the macroscale model we observed that the simulation results with the interface conditions derived by homogenisation (profiles: SD (homogen. A) and SD (homogen. B)) fit very well to the LBM simulation results. For such flow problems, we advise to use condition (12)(14) instead of trying to find the optimal value for $\alpha_{\mathrm{BJ}}$ and to determine the correct interface location.

We observed that the interface condition for the $x_{2}$ component of the velocity, given by Eq. (12), is nevertheless reasonable for such flow problems.

\subsubsection{Staggered geometrical configuration}

Since the Beavers-Joseph parameter $\alpha_{\mathrm{BJ}}$ contains the information about the geometry of the interfacial region, we consider another geometrical configuration for the porous bed. In this case, the porous medium consists of solid inclusions that are arranged in a periodic but staggered manner (Fig. 13). The porosity is kept the same as for the channelised setting $\phi=0.4$. The number of solid inclusions is also the same: 40 inclusions in $x_{1}$-direction and 20 inclusions in $x_{2}$-direction.

For this porous-medium geometry, we obtain the effective permeability tensor $\mathbf{K}=\varepsilon^{2} \widetilde{\mathbf{K}}$ in the same way as before, using formula (21):

$\widetilde{\mathbf{K}}=\left(\begin{array}{cc}4.147 \cdot 10^{-4} & 0 \\ 0 & 1.325 \cdot 10^{-4}\end{array}\right)$,

where $\varepsilon=1 / 20$ and the unit cell $Y$ contains several solid inclusions (Fig. 13, right).

We analysed the macroscale problem (1), (2), (4), (5) with the classical interface conditions (7), (8), (11) and the appropriate boundary conditions at the external boundary.
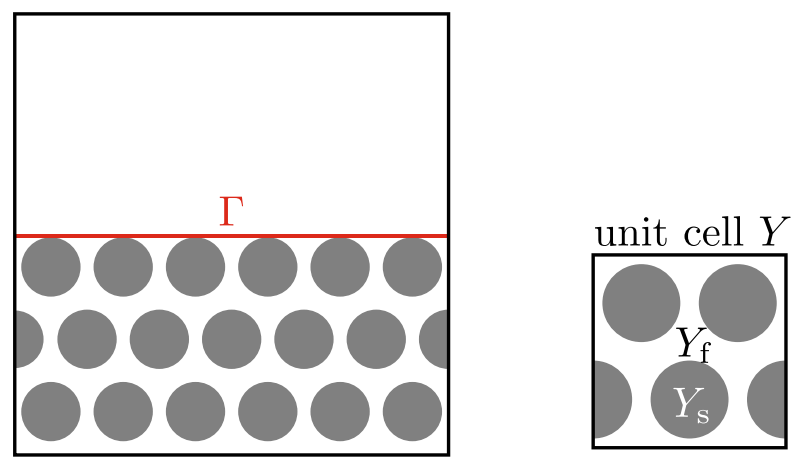

Fig. 13 Schematic illustration of the microscale setting and the location of the sharp fluid-porous interface $\Gamma$ (left) and the unit cell (right) for the staggered lid-driven cavity and the infiltration problem 
Cross-section at $x_{1}=0.5(s=0)$

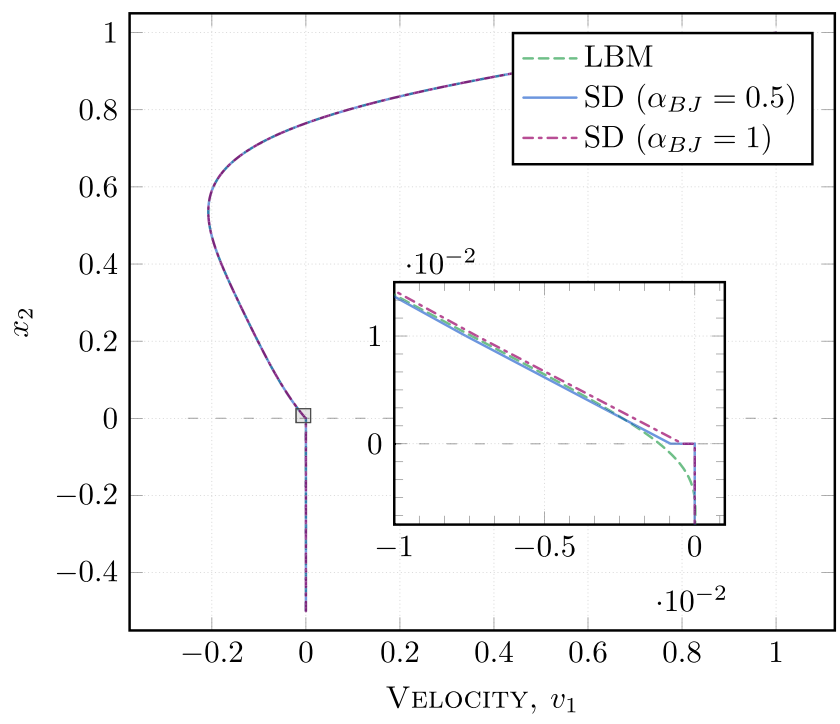

Fig. 14 Velocity profiles ( $x_{1}$-component) for the staggered lid-driven case at $x_{1}=0.5$ for classcial interface conditions zoomed near the interface

Figure 14 provides velocity profiles of the horizontal component of the velocity in the middle of the domain. Again, as we observed for the channelised geometry (Fig. 7), the macroscale simulation results with $\alpha_{\mathrm{BJ}}=0.5$ fit better to the pore-scale resolved simulations than the results with $\alpha_{\mathrm{BJ}}=1$ that is commonly used in the literature.

\subsection{Infiltration}

For the infiltration problem we consider only the staggered geometrical configuration of the porous medium (Fig. 13) to avoid a channel flow in the vertical direction between columns of solid inclusions (Fig. 4). Therefore, the permeability tensor is identical with the one given in Eq. (33).

The boundary conditions for the macroscale model given by equations (1), (2), (4), (5), (7), (8), (11) for the infiltration problem read

$$
\begin{array}{ll}
\mathbf{v}_{\mathrm{ff}}=\left(0,-0.1 \sin \left(\pi x_{1}\right)\right) & \text { on }\left\{x_{2}=1\right\}, \\
\mathbf{v}_{\mathrm{ff}}=(0,0) & \text { on }(\{0\} \cup\{1\}) \times(0,1), \\
p_{\mathrm{pm}}=100 & \text { on }\left\{x_{2}=-0.5\right\}, \\
\mathbf{v}_{\mathrm{pm}} \cdot \mathbf{n}_{\mathrm{pm}}=0 & \text { on }(\{0\} \cup\{1\}) \times(-0.5,0) .
\end{array}
$$

Here, we define the Reynolds number

$\operatorname{Re}=\frac{v_{\mathrm{in}, \max } \mathcal{L}}{v}$,

where $v_{\text {in,max }}$ is the absolute maximum value of the inflow velocity ( $x_{2}$-component). Similar as for the lid-driven cavity problem (Section 6.1), in the LBM simulation, the whole domain is resolved by $2400 \times 3600$ lattice cells. The solid inclusions have a diameter of around 52 lattice cells. To resemble (34), we use simple bounce back boundary conditions at the domain-boundaries in $x_{1}$-direction, at the inflow and at the solid inclusions. At the bottom of the domain in $x_{2}$-direction, we impose pressure boundary conditions based on the anti-bounce back approach [40] and set the lattice density to $\rho=1$. Again, $R e=0.01$ is chosen in order to approximate Stokes flow.

We make cross-sections for the velocity and pressure in the horizontal centre of the domain at $x_{1}=0.5$ and consider different locations of the sharp fluid-porous interface $\Gamma$ for the macroscale model with the classical set of interface conditions. As in Section 6.1, the sharp interface is located at $x_{2}=-0.01(s=b), x_{2}=0(s=0)$ and $x_{2}=0.01$ $(s=t)$.

In Fig. 15 we present the vertical component of the velocity computed for the macroscale model (SD) and the LBM simulations. We normalised the velocity by its maximal absolute value $v_{\text {in,max }}=0.1$. In this case, the macroscale simulation results agree very well to the results of the pore-scale resolved models even though the interface conditions (7), (8), (11) developed for parallel flows were used. This is due to the fact that the $x_{1}$-component of the velocity is almost zero. Therefore, we do not provide the profiles for the horizontal velocity component.

For the infiltration problem, we observed that the pressure field is very sensitive to the location of the sharp interface (Fig. 16). Again, we obtain the best fit of the macroscale to the pore-scale model (Fig. 16, $s=0$ ) for the interface located directly on top of the first row of solid inclusions (Fig. 13). However, the differences between

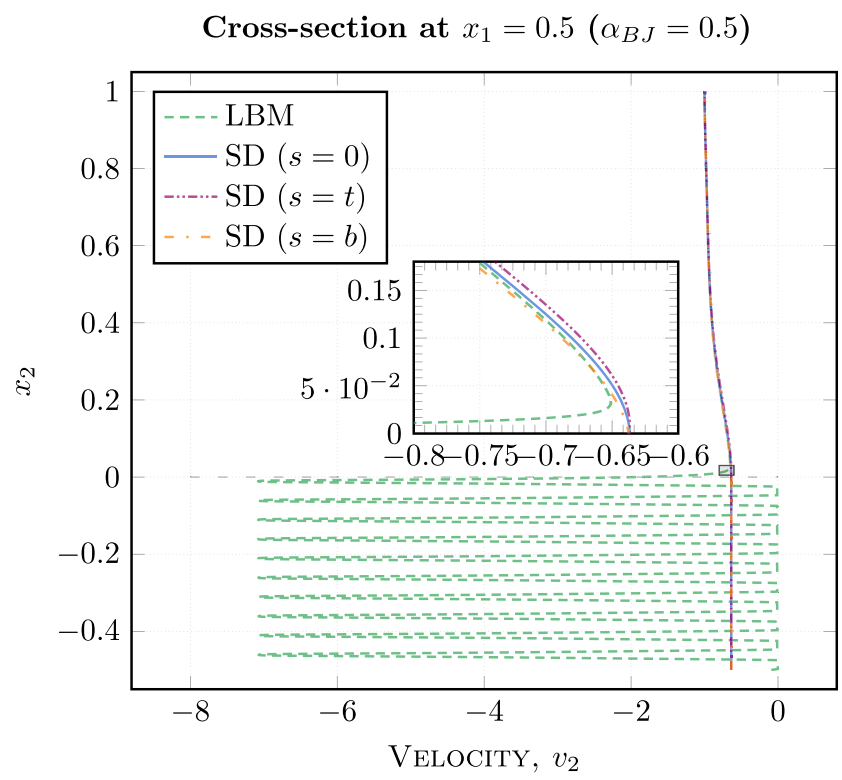

Fig. 15 Velocity profiles ( $x_{2}$-component) for the infiltration problem at $x_{1}=0.5$ zoomed near the interface 
Cross-section at $x_{1}=0.5\left(\alpha_{B J}=0.5\right)$

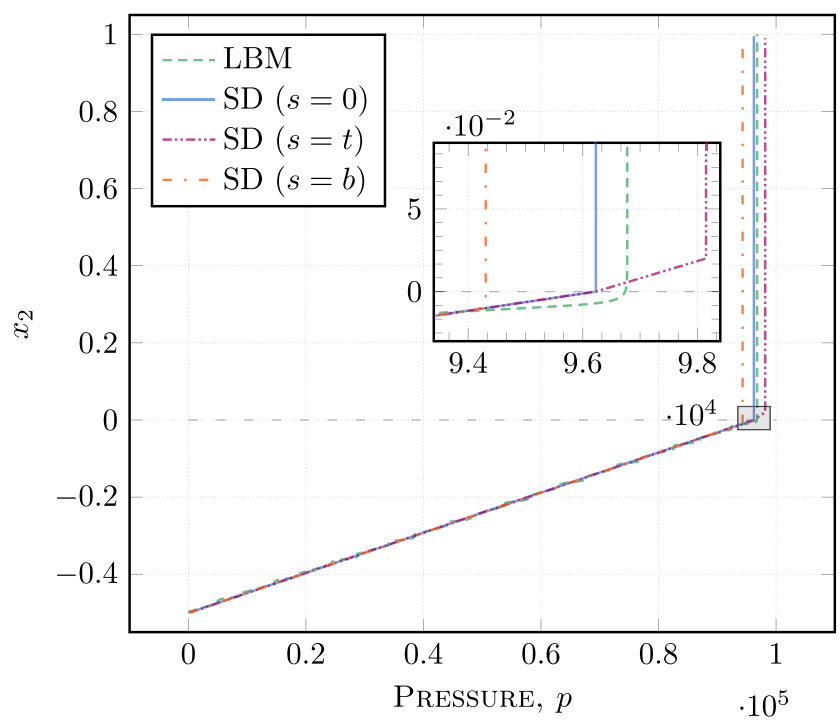

Fig. 16 Pressure profiles for the infiltration problem at $x_{1}=0.5$ for different interface locations zoomed near the interface

the pressure field obtained from the LBM simulations and the one from the best fitting interface location of the macroscopic model are more significant compared with the differences we observed for the velocity profiles.

\subsection{General filtration problem}

In this section, we demonstrate that the classical interface conditions (7), (8), (11) and the interface conditions based on homogenisation (12)-(14) are unsuitable for modelling coupled problems with arbitrary flows to the interface. A detailed study can be found in [33].

We consider a channelised geometrical configuration of the porous medium (Fig. 4) with $20 \times 10$ solid inclusions, thus $\varepsilon=1 / 20$. The radius of the solid obstacles is $r=0.25 \varepsilon$ and the porosity in this case is $\phi \approx 0.8$. The permeability tensor reads

$\tilde{\mathbf{K}}=\left(\begin{array}{cc}1.999 \cdot 10^{-2} & 0 \\ 0 & 1.999 \cdot 10^{-2}\end{array}\right)$

Again, to obtain the effective permeability tensor, we scale $\mathbf{K}=\varepsilon^{2} \widetilde{\mathbf{K}}$. For this geometry and conditions (12)-(14) we set the interface $\Gamma$ at $x_{2}=1.25 \cdot 10^{-2}$ and obtain the following boundary layer constants

$C_{1}^{\mathrm{bl}}=-3.00 \cdot 10^{-1}, \quad C_{\omega}^{\mathrm{bl}}=0$.
The boundary conditions for the macroscale model in this case read

$$
\begin{array}{ll}
\mathbf{v}_{\mathrm{ff}}=\left(0,-0.2 \sin \left(\pi x_{1}\right)\right) & \text { on }\left\{x_{2}=H\right\}, \\
\left(\nabla \mathbf{v}_{\mathrm{ff}}-p_{\mathrm{ff}} \mathbf{I}\right) \mathbf{n}=\mathbf{0} & \text { on } \Gamma_{\mathrm{r}, \text { out }} \cup \Gamma_{\mathrm{l}, \text { out }}, \\
\mathbf{v}_{\mathrm{ff}}=(0,0) & \text { on } \Gamma_{\mathrm{wall}} \cap\left\{x_{2}>0\right\}, \\
\mathbf{v}_{\mathrm{pm}} \cdot \mathbf{n}=0 & \text { on } \Gamma_{\mathrm{wall}} \cap\left\{x_{2}<0\right\}, \\
p_{\mathrm{pm}}=\left(10^{-6}-x_{1}\right) & \text { on }\left\{x_{2}=h\right\},
\end{array}
$$

where $\Gamma_{\mathrm{l}, \text { out }}, \Gamma_{\mathrm{r} \text {,out }}$ and $\Gamma_{\mathrm{wall}}$ are defined in Section 4.1. We have $R e=0.01$ as defined in (35) in order to approximate Stokes flow. In the LBM simulations, the whole domain is resolved by $2400 \times 2400$ lattice cells such that solid inclusions have a diameter of about 60 lattice cells. Solid inclusions, solid domain walls and the inflow are modelled by bounce back boundary conditions. The outflows are realised with anti-bounce back pressure boundary conditions. Here, the LBM density is set to $\rho=1$ at $\Gamma_{\text {r,out }} \cup \Gamma_{1, \text { out }}$ and $\rho=1+7.23^{-10} \cdot p_{\text {pm }}$ at $x_{2}=h$ with $7.23^{-10}$ being the unit conversion factor for converting the macroscale model's pressure to the density in LBM's lattice units.

In Fig. 17 we provide the profiles of the horizontal velocity component at $x_{1}=0.2$, where the flow is arbitrary to the interface. We normalised the velocity by its maximal absolute value at the top boundary $v_{\text {in,max }}=0.2$. We consider different values of parameter $\alpha_{\mathrm{BJ}}$ for the classical interface conditions (7), (8), (11). In case of the conditions derived using homogenisation (12)-(14) the boundary layer constants (37) are computed based on the pore geometry. Evidently, none of the macroscale

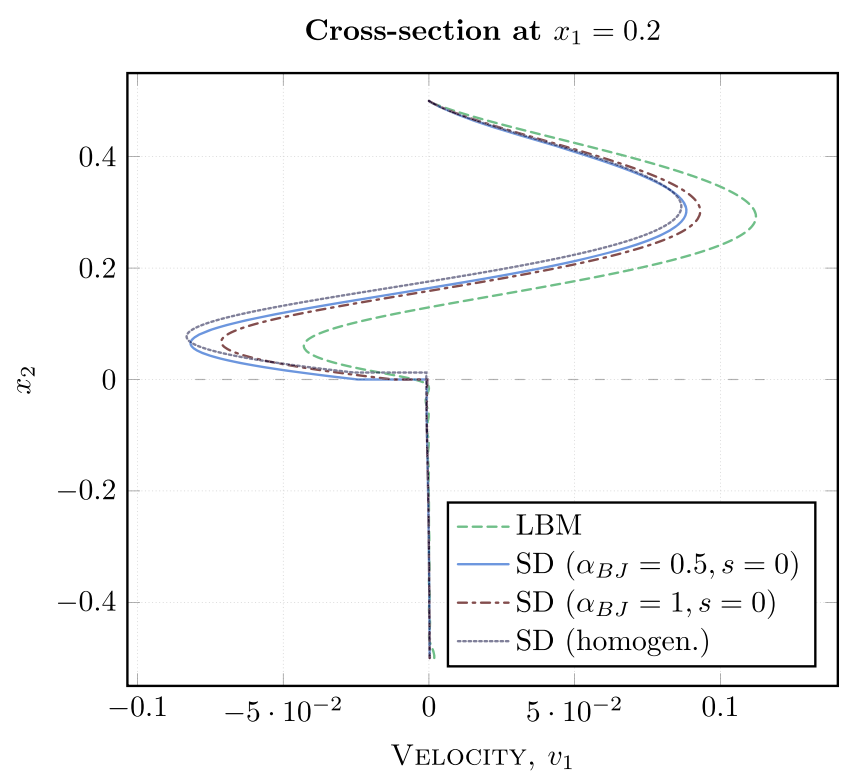

Fig. 17 Velocity profiles ( $x_{1}$-component) at $x_{1}=0.2$ for the general filtration problem 
simulation results agree with the pore-scale resolved results. This test case demonstrates that neither the classical coupling conditions (7), (8), (11) nor the conditions based on homogenisation (12)-(14) are suitable for arbitrary flows to the interface. Therefore, new interface conditions are needed in order to describe general fluid flows in coupled systems.

\section{Conclusions}

In this paper, we validated two sets of interface conditions for the coupled Stokes-Darcy problem: (i) the classical conservation of mass across the interface, the balance of normal forces and the Beavers-Joseph-Saffman interface condition, and (ii) the interface conditions derived by means of the homogenisation and boundary layer theory [11, 36]. We considered different geometrical configurations (channelised and staggered arrangement of solid grains) and different flow problems (lid-driven cavity over a porous bed, infiltration and general filtration problem). The effective model parameters (permeability, boundary layer constants) are computed numerically for the geometrical settings examined in the paper. To be able to compute these parameters using homogenisation theory, we considered periodic porous media. The interface conditions are validated and calibrated numerically by comparing macroscale simulation results against pore-scale simulations. As a pore-scale resolved model we used large-scale lattice Boltzmann simulations.

Numerical simulation results demonstrate sensitivity of the coupled Stokes-Darcy problem to the choice of the model parameter $\alpha_{\mathrm{BJ}}$, the location of the sharp interface and the choice of interface conditions. We have tested a number of characteristic flow regimes in low Reynolds number cases and observed that: i) for flows parallel to the porous medium $\alpha_{\mathrm{BJ}}=0.5$ seems to be the best candidate for the Beavers-Joseph parameter and therefore, the commonly used value $\alpha_{\mathrm{BJ}}=1$ is often not the optimal choice; ii) for flows perpendicular to the interface, the Beavers-JosephSaffman condition (11) can be applied and the choice of parameter $\alpha_{\mathrm{BJ}}$ is not important here due to small horizontal velocity; iii) for arbitrary flows to the interface neither the classical conditions (7), (8), (11) nor the conditions based on homogenisation (12)-(14) are suitable. Moreover, iv) the sharp fluid-porous interface should be located directly on the top of the first row of solid inclusions for the classical interface conditions; and $\mathrm{v}$ ) for flows parallel to the porous medium, we suggest to use the interface conditions derived by homogenisation (12)-(14) since in this case all model parameters can be computed numerically based on the pore geometry and the chosen interface location. Thus, no parameter fitting is needed.
Acknowledgements Open Access funding provided by Projekt DEAL. The authors thank Ivan Yotov, Jim Magiera and Christoph Rettinger for the valuable discussions. The authors are grateful to the Regionales Rechenzentrum Erlangen (www.rrze.de) for providing access to supercomputing facilities.

Funding I. Rybak and E. Eggenweiler received financial support for this work from the Deutsche Forschungsgemeinschaft (DFG, German Research Foundation) by funding SFB 1313, Project Number 327154368. C. Schwarzmeier and U. Rüde received funding from the DFG for supporting project RU 422/27 and the Bundesministerium für Bildung und Forschung (BMBF, Federal Ministry of Education and Research) for supporting the projects HPC2SE (01ICH16003D) and SKAMPY (01IH15003A).

Open Access This article is licensed under a Creative Commons Attribution 4.0 International License, which permits use, sharing, adaptation, distribution and reproduction in any medium or format, as long as you give appropriate credit to the original author(s) and the source, provide a link to the Creative Commons licence, and indicate if changes were made. The images or other third party material in this article are included in the article's Creative Commons licence, unless indicated otherwise in a credit line to the material. If material is not included in the article's Creative Commons licence and your intended use is not permitted by statutory regulation or exceeds the permitted use, you will need to obtain permission directly from the copyright holder. To view a copy of this licence, visit http:// creativecommonshorg/licenses/by/4.0/.

\section{References}

1. Blunt, M.J.: Multiphase flow in permeable media: A pore-scale perspective. Cambridge University Press, Cambridge (2017)

2. Wildenschild, D., Sheppard, A.P.: X-ray imaging and analysis techniques for quantifying pore-scale structure and processes in subsurface porous medium systems. Adv. Water Res. 51, 217-246 (2013)

3. Beavers, G.S., Joseph, D.D.: Boundary conditions at a naturally permeable wall. J. Fluid Mech. 30, 197-207 (1967)

4. Goyeau, B., Lhuillier, D., Gobin, D., Velarde, M.: Momentum transport at a fluid-porous interface. Int. J. Heat Mass Transfer 46 , 4071-4081 (2003)

5. Nield, D.A.: The Beavers-Joseph boundary condition and related matters: a historical and critical note. Transp. Porous Media 78, 537-540 (2009)

6. Ochoa-Tapia, A.J., Whitaker, S.: Momentum transfer at the boundary between a porous medium and a homogeneous fluid. I: Theoretical development. Int. J. Heat Mass Transfer 38, 26352646 (1995)

7. Saffman, P.G.: On the boundary condition at the surface of a porous medium. Stud. Appl. Math. 50, 93-101 (1971)

8. Discacciati, M., Miglio, E., Quarteroni, A.: Mathematical and numerical models for coupling surface and groundwater flows. Appl. Num. Math. 43, 57-74 (2002)

9. Discacciati, M., Quarteroni, A.: Navier-Stokes/Darcy coupling: modeling, analysis, and numerical approximation. Rev. Mat. Complut. 22, 315-426 (2009)

10. Jackson, A.S., Rybak, I., Helmig, R., Gray, W.G., Miller, C.T.: Thermodynamically constrained averaging theory approach for modeling flow and transport phenomena in porous medium systems: 9. Transition region models. Adv. Water Res. 42, 71-90 (2012) 
11. Jäger, W., Mikelić, A.: Modeling effective interface laws for transport phenomena between an unconfined fluid and a porous medium using homogenization. Transp. Porous Media 78, 489508 (2009)

12. Layton, W., Schieweck, F., Yotov, I.: Coupling fluid flow with porous media flow. SIAM J. Numer. Anal. 40, 2195-2218 (2003)

13. Dawson, C.: A continuous/discontinuous Galerkin framework for modeling coupled subsurface and surface water flow. Comput. Geosci. 12, 451-472 (2008)

14. Sochala, P., Ern, A., Piperno, S.: Mass conservative BDFdiscontinuous Galerkin/explicit finite volume schemes for coupling subsurface and overland flows. Comput. Methods Appl. Mech. Engrg. 198, 2122-2136 (2009)

15. Reuter, B., Rupp, A., Aizinger, V., Knabner, P.: Discontinuous Galerkin method for coupling hydrostatic free surface flows to saturated subsurface systems. Comput. Math. Appl. 77, 22912309 (2019)

16. Brinkman, H.C.: A calculation of the viscous force exerted by a flowing fluid on a dense swarm of particles. Appl. Sci. Res. 1, 27 34 (1947)

17. Cimolin, F., Discacciati, M.: Navier-Stokes/Forchheimer models for filtration through porous media. Appl. Numer. Math. 72, 205224 (2013)

18. Mosthaf, K., Baber, K., Flemisch, B., Helmig, R., Leijnse, A., Rybak, I., Wohlmuth, B.: A coupling concept for two-phase compositional porous-medium and single-phase compositional free flow. Water Resour. Res. 47, W10522 (2011)

19. Rybak, I., Magiera, J., Helmig, R., Rohde, C.: Multirate time integration for coupled saturated/unsaturated porous medium and free flow systems. Comput. Geosci. 19, 299-309 (2015)

20. Carraro, T., Goll, C., Marciniak-Czochra, A., Mikelić, A.: Effective interface conditions for the forced infiltration of a viscous fluid into a porous medium using homogenization. Comput. Methods Appl. Mech. Engrg. 292, 195-220 (2015)

21. Angot, P., Goyeau, B., Ochoa-Tapia, J.A.: Asymptotic modeling of transport phenomena at the interface between a fluid and a porous layer: Jump conditions. Phys. Rev. E 95, 063302 (2017)

22. Arbogast, T., Brunson, D.S.: A computational method for approximating a Darcy-Stokes system governing a vuggy porous medium. Comput. Geosci. 11, 207-218 (2007)

23. Hanspal, N.S., Waghode, A.N., Nassehi, V., Wakeman, R.J.: Numerical analysis of coupled Stokes/Darcy flows in industrial filtrations. Transp. Porous Media 64, 383-411 (2006)

24. Iliev, O., Laptev, V.: On numerical simulation of flow through oil filters. Comput. Vis. Sci. 6, 139-146 (2004)

25. Rivière, B.: Analysis of a discontinuous finite element method for the coupled Stokes and Darcy problems. J. Sci. Comput. 22, 479500 (2005)

26. Girault, V., Rivière, B.: DG approximation of coupled NavierStokes and Darcy equations by Beavers-Joseph-Saffman interface condition. SIAM J. Numer. Anal. 47, 2052-2089 (2009)

27. Jäger, W., Mikelić, A.: On the interface boundary conditions by Beavers, Joseph and Saffman. SIAM J. Appl. Math. 60, 11111127 (2000)

28. Valdés-Parada, F.J., Alvarez-Ramìrez, J., Goyeau, B., OchoaTapia, J.A.: Computation of jump coefficients for momentum transfer between a porous medium and a fluid using a closed generalized transfer equation. Transp. Porous Media 78, 439-457 (2009)

29. Lācis, U., Bagheri, S.: A framework for computing effective boundary conditions at the interface between free fluid and a porous medium. J. Fluid Mech. 812, 866-889 (2017)
30. Zampogna, G.A., Bottaro, A.: Fluid flow over and through a regular bundle of rigid fibres. J. Fluid Mech. 792, 5-35 (2016)

31. Le Bars, M., Worster, M.: Interfacial conditions between a pure fluid and a porous medium: implications for binary alloy solidification. J. Fluid Mech. 550, 149-173 (2006)

32. Yang, G., Coltman, E., Weishaupt, K., Terzis, A., Helmig, R., Weigand, B.: On the Beavers-Joseph interface condition for nonparallel coupled channel flow over a porous structure at high Reynolds numbers. Transp. Porous Media 128, 431-457 (2019)

33. Eggenweiler, E., Rybak, I.: Unsuitability of the Beavers-Joseph interface condition for filtration problems. J. Fluid Mech. 892, A10 (2020)

34. Discacciati, M., Gerardo-Giorda, L.: Optimized Schwarz methods for the Stokes-Darcy coupling. IMA J. Numer. Anal. 38, 19591983 (2018)

35. Jones, I.P.: Low Reynolds number flow past a porous spherical shell. Proc. Camb. Phil. Soc. 73, 231-238 (1973)

36. Jäger, W., Mikelić, A., Neuss, N.: Asymptotic analysis of the laminar viscous flow over a porous bed. SIAM J. Sci. Comput. 22, 2006-2028 (2001)

37. Hornung, U.: Homogenization and porous media. Springer, Berlin (1997)

38. Wolf-Gladrow, D.: Lattice-gas cellular automata and lattice Boltzmann models. Springer, Berlin (2000)

39. He, X., Luo, L.-S.: A priori derivation of the lattice Boltzmann equation. Phys. Rev. E 55, R6333 (1997)

40. Krüger, T., Kusumaatmaja, H., Kuzmin, A., Shardt, O., Silva, G., Viggen, E.M.: The lattice Boltzmann method: Principles and practice. Springer, Berlin (2017)

41. Rettinger, C., Godenschwager, C., Eibl, S., Preclik, T., Schruff, T., Frings, R., Rüde, U.: Fully resolved simulations of dune formation in riverbeds. In: Proceedings of the International Conference on High Performance Computing, pp. 3-21. Springer International Publishing (2017)

42. Kuron, M., Rempfer, G., Schornbaum, F., Bauer, M., Godenschwager, C., Holm, C., de Graaf, J.: Moving charged particles in lattice Boltzmann-based electrokinetics. J. Chem. Phys. 145(21), 214102 (2016)

43. Huang, H., Sukop, M.C., Lu, X.-Y.: Multiphase lattice Boltzmann methods: Theory and application. Wiley, New York (2015)

44. Fattahi, E., Waluga, C., Wohlmuth, B., Rüde, U.: Large scale lattice Boltzmann simulation for the coupling of free and porous media flow, pp. 1-18. Springer (2016)

45. Fattahi, E., Waluga, C., Wohlmuth, B., Rüde, U., Manhart, M., Helmig, R.: Lattice Boltzmann methods in porous media simulations: From laminar to turbulent flow. Comput. Fluids 140, 247-259 (2016)

46. Pan, C., Luo, L., Miller, C.T.: An evaluation of lattice Boltzmann schemes for porous medium flow simulation. Comput. Fluids 35, 898-909 (2006)

47. Ginzburg, I., Verhaeghe, F., d'Humières, D.: Two-relaxationtime lattice Boltzmann scheme: About parametrization, velocity, pressure and mixed boundary conditions. Commun. Comput. Phys. 3, 427-478 (2008)

48. Ginzburg, I., Verhaeghe, F., d'Humières, D.: Study of simple hydrodynamic solutions with the two-relaxation-times lattice Boltzmann scheme. Commun. Comput. Phys. 3, 519-581 (2008)

49. Hecht, F.: New development in FreeFem++. J. Numer. Math. 20, 251-265 (2012)

50. Bauer, M., Eibl, S., Godenschwager, C., Kohl, N., Kuron, M., Rettinger, C., Schornbaum, F., Schwarzmeier, C., Thönnes, D., Köstler, H., Rüde, U.: waLBerla: A block-structured 
high-performance framework for multiphysics simulations. CAMWA (2020)

51. Godenschwager, C., Schornbaum, F., Bauer, M., Köstler, H., Rüde, U.: A framework for hybrid parallel flow simulations with a trillion cells in complex geometries. In: Proceedings of the International Conference on High Performance Computing, Networking, Storage and Analysis, pp. 1-12. ACM (2013)
52. Schornbaum, F., Rüde, U.: Extreme-scale block-structured adaptive mesh refinement. SIAM J. Sci. Comput. 40(3), C358-C387 (2018)

Publisher's note Springer Nature remains neutral with regard to jurisdictional claims in published maps and institutional affiliations.

\section{Affiliations}

\section{Iryna Rybak $^{1}$ (D) . Christoph Schwarzmeier ${ }^{2}$ - Elissa Eggenweiler ${ }^{1}$ - Ulrich Rüde $^{2,3}$}

Christoph Schwarzmeier

christoph.schwarzmeier@fau.de

Elissa Eggenweiler

elissa.eggenweiler@mathematik.uni-stuttgart.de

Ulrich Rüde

ulrich.ruede@fau.de

1 Institute of Applied Analysis and Numerical Simulation, University of Stuttgart, Pfaffenwaldring 57, 70569,

Stuttgart, Germany

2 Chair for System Simulation, Friedrich-Alexander University Erlangen-Nürnberg, Cauerstraße 11, 91058, Erlangen, Germany

3 CERFACS, 42 Avenue Gaspard Coriolis, 31057, Toulouse, France 